\title{
Aura of technology and the cutting edge: a history of lasers in neurosurgery
}

\author{
Robert W. Ryan, M.D., Robert F. Spetzler, M.D., and Mark C. Preul, M.D. \\ Neurosurgery Research Laboratory, Division of Neurological Surgery, Barrow Neurological Institute, \\ St. Joseph's Hospital and Medical Center, Phoenix, Arizona
}

\begin{abstract}
In this historical review the authors examine the important developments that have led to the availability of laser energy to neurosurgeons as a unique and sometimes invaluable tool. They review the physical science behind the function of lasers, as well as how and when various lasers based on different lasing mediums were discovered. They also follow the close association between advances in laser technology and their application in biomedicine, from early laboratory experiments to the first clinical experiences. Because opinions on the appropriate role of lasers in neurosurgery vary widely, the historical basis for some of these views is explored. Initial enthusiasm for a technology that appears to have innate advantages for safe resections has often given way to the strict limitations and demands of the neurosurgical operating theater. However, numerous creative solutions to improve laser delivery, power, safety, and ergonomics demonstrate the important role that technological advances in related scientific fields continue to offer neurosurgery. Benefiting from the most recent developments in materials science, current $\mathrm{CO}_{2}$ laser delivery systems provide a useful addition to the neurosurgical armamentarium when applied in the correct circumstances and reflect the important historical advances that come about from the interplay between neurosurgery and technology. (DOI: 10.3171/2009.6.FOCUS09125)
\end{abstract}

KEY WORDS • laser surgery $\quad$ - neurosurgical procedure $\quad$ neurosurgical history

$\mathrm{T}$ HE association of technology with neurosurgery has created perhaps the most advanced integration of any medical specialty, from diagnostic techniques to operative guidance systems, surgical equipment, and monitoring. To many patients and laypeople, the combination of brain surgery and the battery of high-tech adjuncts can be overwhelming, but their desire is the same: to receive the best possible care with the aid of the best available tools. In light of the mystique surrounding neurosurgical procedures and the instruments required to perform them, perhaps it is not surprising that some patients, hoping to benefit from the newest and best, ask whether the laser will be used for their operation. Although in many cases there is no role for such a device, the history of the use of lasers in neurosurgery reveals ingenuity in diverse settings, taking advantage of the individual properties of different laser types. In certain cases, lasers remain a distinct and important addition to the neurosurgical armamentarium.

\section{Basic Laser Physics}

"Laser" is an acronym for "light amplification by stimulated emission of radiation." A simple laser consists

Abbreviation used in this paper: $\mathrm{Nd}: \mathrm{YAG}=$ neodymium:yttrium aluminum garnet. of a lasing medium enclosed between 2 mirrors, 1 of which is able to partially transmit light. When energy is applied to the lasing medium, more atoms of the medium attain an excited state. A photon that strikes an atom in the excited state will cause it to fall to a lower energy state and release a photon of the same wavelength, traveling in the same direction and in phase with the first photon-this process is stimulated emission. The coherence of laser light allows it to be focused into intense beams that can be used in many different applications. ${ }^{14}$

Surgical lasers are typically applied for 3 functions: photocoagulation, to control bleeding and devitalize tissue; photovaporization, to incise and vaporize tissue; and photoactivation, to sensitize tissue or activate photoreactive drugs. The properties of both the laser and the affected tissue are important in determining laser-tissue interactions. Laser properties include wavelength (determined by the lasing medium, such as $\mathrm{CO}_{2}$ ), power output (watts), beam density (spot size), and time of exposure. Together, these factors determine the energy density (W/ $\mathrm{cm}^{2} \times$ time) delivered to the tissue. Important properties inherent to the tissue itself include the absorption coefficient (absorbed light is rapidly converted to heat), the extinction length (the depth that light will penetrate), and the presence of light-absorbing chromophores such as water, hemoglobin, or melanin. ${ }^{9}$ Taken together, laser and tissue factors determine the rate of tissue heating, the area of laser effect, and the type of lesion created. 


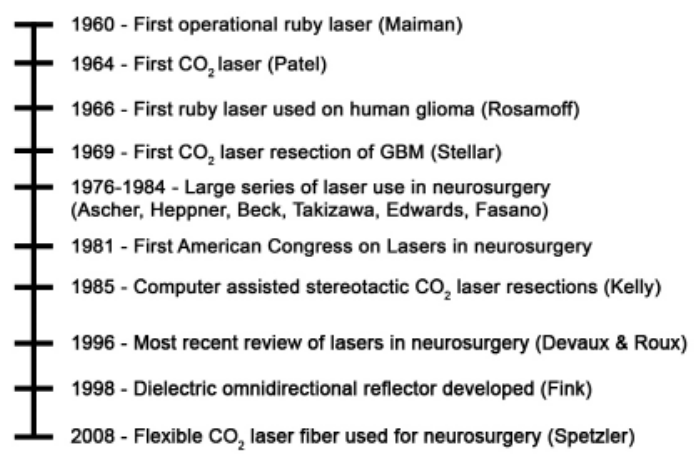

FIG. 1. A timeline listing important events in the use of lasers in neurosurgery. GBM = glioblastoma multiforme.

\section{Discovery of Lasers and Early Animal Experiments}

A timeline of important events in the use of lasers in neurosurgery appears in Fig. 1. The first operational laser was described by Maiman ${ }^{22}$ in 1960 , while working at Hughes Research Laboratories; he used a ruby medium to produce a laser pulse with a 694-nm wavelength. The next reported laser, based in a helium-neon gas medium, was described in 1961 by Javan et al. ${ }^{19}$ from Bell Laboratories in New Jersey. A number of other lasers using gas and solid-state media were developed: importantly, a molecular laser using $\mathrm{CO}_{2}$ as the medium and producing a continuous wave laser at a wavelength of $10.6 \mu \mathrm{m}$, which was described in 1964 by Patel, ${ }^{26}$ who was also working at Bell Laboratories. The early use of the laser for industrial or military applications was reflected in much of the research performed in nonacademic settings. Nonetheless, biomedical applications soon followed, and

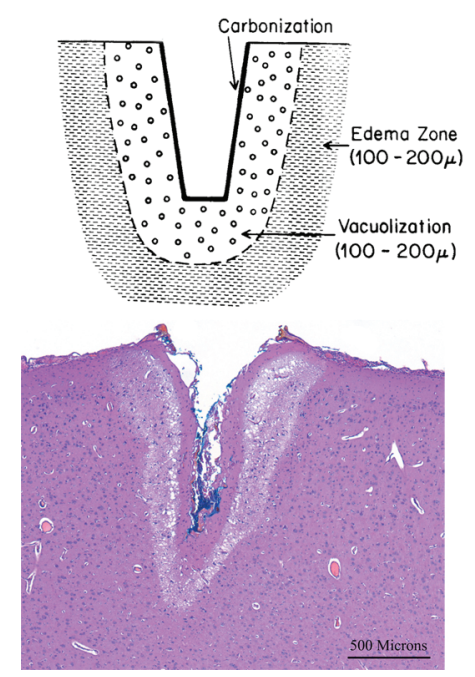

FIG. 2. A schematic (upper) demonstrating the appearance of a typical laser incision in the cortex with 3 zones: a central crater with a carbonized border from high temperature, a middle zone of desiccated tissue from moderate heating, and an outer zone of edematous tissue from minimal heating. Reprinted with permission of John Wiley \& Sons, Inc., from Jain KK: Lasers in neurosurgery: a review. Lasers Surg Med 2:21-230, 1983. Photomicrograph (lower) of a stained section of a typical $\mathrm{CO}_{2}$ laser lesion, revealing the same 3 zones as in the drawing. $\mathrm{H} \& \mathrm{E}$.

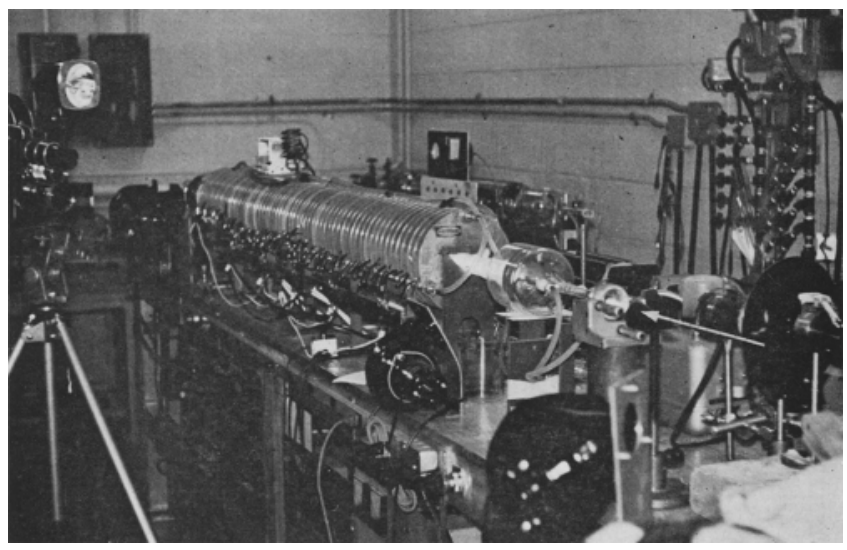

FIG. 3. Photograph of an early argon continuous-wave laser. White arrow indicates the beam emitter. The large size of the unit made it impractical for clinical or research applications, as targets had to be moved into the path of the beam, although it did demonstrate the more controllable nature of continuous-wave laser energy, and therefore more usable delivery systems were developed. Reproduced with permission from Fox et al: J Neurosurg 27:126-137, 1967.

the first reported application of laser energy to the brain was detailed in 1965 in the experiments by Earle et al. ${ }^{8}$ and Fine et al. ${ }^{11}$ These authors applied a single high-energy pulse of a ruby laser to the intact craniums of mice and observed that the mice were instantly killed, their deaths resulting from rapid, explosive expansion of the intracranial contents and cerebral herniation. Other early investigators using pulsed lasers similarly found them to be destructive to neural tissue; however, death from herniation could be prevented by widely exposing the cortex, and this allowed the study of laser-tissue interactions in surviving animals, including observation of sharply demarcated, wedge-shaped lesions. ${ }^{13,29}$ Furthermore, the reproducibility of the injuries resulting from pulsed lasers, including subdural and subarachnoid hemorrhage and underlying cerebral edema, led Brown et al. ${ }^{4}$ to develop a model for studying these pathologies in rats. When lasers were applied at low power in extracranial animal tumor models in 1964, 2 research groups found that pulsed laser energy appeared to have a selective effect, destroying cancer cells, and this result led to hope for similar benefits for oncological treatment elsewhere in the body. ${ }^{23,24}$ Thus the initial findings regarding pulsed-wave lasers applied to the brain suggested that while useful as a research tool, the traumatic results of high-energy pulses and the unpredictable effects of low-energy pulses appeared to limit their utility in clinical neurosurgery.

A major advance in making lasers more applicable to neurosurgery came with the introduction of continuouswave lasers and improved delivery systems. This type of laser energy eliminated the explosive effects of pulsedwave lasers and allowed accurate cutting and vaporization by using focused beams, without the need to handle or retract the tissue. ${ }^{13}$ Investigators also began to take advantage of the fundamental properties of different types of lasers. The long wavelength of the $\mathrm{CO}_{2}$ laser $(10.6 \mu \mathrm{m})$ was found to have high absorption in tissue and water, with rapid conversion of light energy into heat in a small volume of tissue. ${ }^{16}$ This profile makes the $\mathrm{CO}_{2}$ laser an 


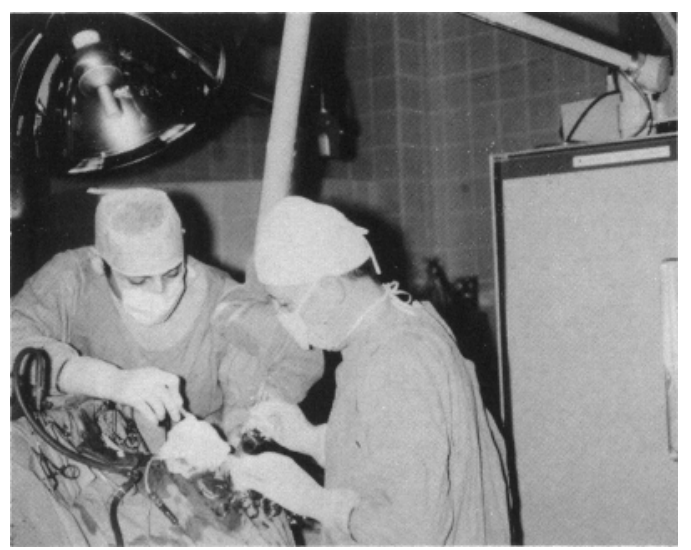

FIG. 4. Photograph of Drs. S. Stellar (right) and K. Samra performing the first resection of a GBM using a $\mathrm{CO}_{2}$ laser. Note the large articulating arm on the right side of the image delivering the laser energy to the operative site. Reproduced with permission from Stellar S and Polanyi TJ: Lasers in neurosurgery: a historical overview. J Clin Laser Med Surg 10:399-411, 1992.

excellent cutting tool, as it causes minimal thermal damage to adjacent tissue but limits its ability to coagulate large vessels. Extensive experiments on the lesion characteristics produced by $\mathrm{CO}_{2}$ laser beams were conducted by Stellar et al. ${ }^{32}$ and Ascher, ${ }^{1}$ who described characteristic zones, including a vaporized crater surrounded by a zone of desiccated tissue and an outer zone of edematous tissue (Fig. 2). Stellar and colleagues also investigated the ability of the $\mathrm{CO}_{2}$ laser to vaporize and resect a brain tumor in a mouse model, and their results inspired future clinical applications. ${ }^{32}$

In contrast to the long wavelength of the $\mathrm{CO}_{2}$ laser, the argon laser, with a wavelength of $488-516 \mathrm{~nm}$, is scattered more widely in tissue, creating a broader zone of heating and tissue effect, but is absorbed by hemoglobin, producing effective coagulation. ${ }^{9}$ The ability of argon and $\mathrm{CO}_{2}$ lasers to be delivered at high power as a continuous wave led to the first experiments with controlled laser energy in 1967, but the large setup hindered initial use because of the difficulty in getting the beam to the target (Fig. 3). ${ }^{13}$ Newer delivery systems, including fiberoptic cables for shorter wavelength lasers and articulated arms with reflecting mirrors for longer wavelengths, addressed this problem. The Nd:YAG laser, with a wavelength of 1060-1340 nm, was subsequently found to scatter widely in tissue, producing slow, deep heating with a wide area of effect, which is very useful for coagulation. ${ }^{10}$ Further experiments in the 1980s expanded the use of Nd:YAG lasers for the anastomosis of blood vessels as well as their coagulating and vaporizing abilities, but their wide thermal effects limited use near eloquent structures. ${ }^{18}$

\section{First Clinical Experience}

Inspired by the selective cancer cell destruction reported after exposure to pulsed ruby laser energy, Rosomoff and Carroll ${ }^{30}$ were the first, in 1966, to apply this type of laser to malignant gliomas in humans following standard operative exposure. As the pulse energy needed to be low to avoid explosive damage to adjacent structures,

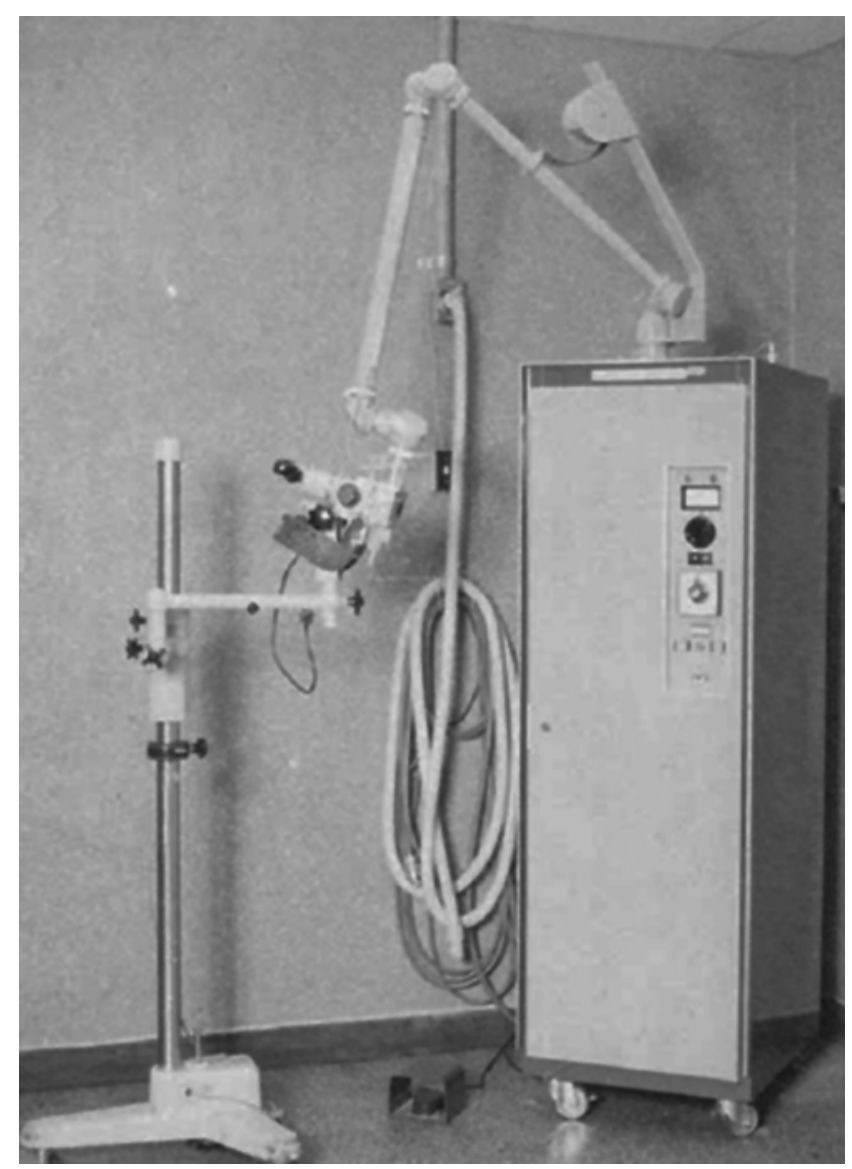

FIG. 5. Photograph of an American Optical Corporation $\mathrm{CO}_{2}$ laser (right) with an articulated arm connected to an operating microscope (left). Note the foot pedal for controlling operation of the laser. Reproduced with permission from John Wiley \& Sons, Inc., from Stellar S: The carbon dioxide surgical laser in neurological surgery, decubitis ulcers and burns. Lasers Surg Med 1:15-33, 1980.

no attempt was made to resect tumor tissue with the laser, and only minimal areas of tumor necrosis were induced, with no improvement in patient survival. Encouraged by their results in animal models, Stellar and colleagues ${ }^{31,32}$ were, in 1969, the first to attempt the resection of a human brain tumor, a recurrent glioblastoma multiforme, by using the continuous-wave $\mathrm{CO}_{2}$ laser (Fig. 4). Given the location of the tumor in the left frontal area, gross-total resection was not attempted, but the patient recovered well from the operation with initial neurological improvement. This result led the authors to state that the use of the $\mathrm{CO}_{2}$ laser for "additional otherwise hopeless human brain tumours is now warranted." Note, however, that logistical issues, including much longer operative times and the cumbersome nature of the articulating arms, which were difficult to use and impeded vision, tempered initial optimism, and few clinical advances took place in the 1970 s.

\section{Growing Enthusiasm}

The ability of the $\mathrm{CO}_{2}$ laser to both cut and coagulate with a no-touch technique and minimal tissue trauma convinced some neurosurgeons to continue to explore its 


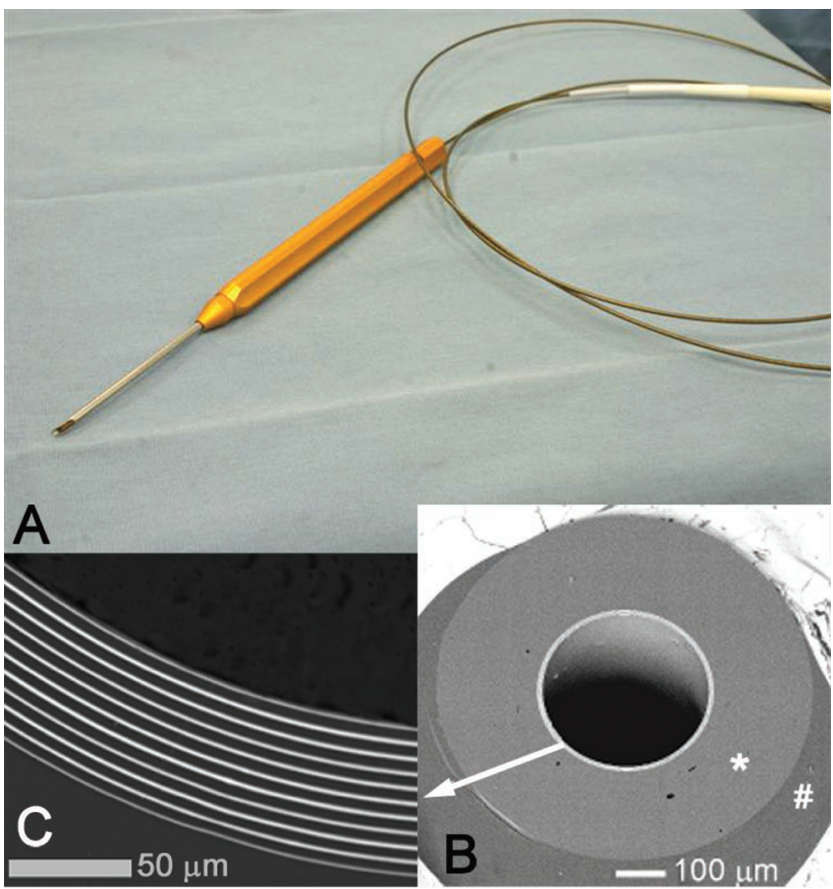

FIG. 6. A: A flexible fiber lined by a dielectric, omnidirectional mirror for transmission of $\mathrm{CO}_{2}$ laser energy (BeamPath Neuro-L fiber, OmniGuide, Inc.) passing through a handheld guide. Note the fiber is slightly recessed in the guide, allowing the distal tip to be used as a nonenergized dissector, and the instrument can be used freely under the microscope. B: Cross-section of the fiber as viewed under a scanning electron microscope. The hollow core (black) is seen at the center of the fiber, surrounded by the layers of mirror (white) that line the core circumferentially. The mirror is embedded in a poly(ether sulfone) fiber cladding (asterisk) and surrounded by epoxy (octothorpe). C: A higher magnification of the mirror layers. This novel architecture allows the propagation of $\mathrm{CO}_{2}$ laser energy through a flexible fiber.

utility, although its use remained anecdotal until several large studies were reported at the end of the 1970s and early 1980s. Perhaps most supportive of the new technology was the Ascher and Heppner ${ }^{2}$ report of 657 cases from 1976 to 1983 that included 546 intracranial tumors, 33 spinal cases, 26 peripheral nerve cases, and 52 other procedures. The authors praised the precise, no-touch cutting of the $\mathrm{CO}_{2}$ laser, which made it easy to extirpate tough tumors, and suggested that gliosis and painful neuroma formation were reduced when the laser was used. Moreover, they identified indications for the use of the laser: "absolute" (all fibrous tumors of the midline, pineal region, brainstem, and spinal cord), "relative" (if either the surgeon or patient receives benefit from using the laser), "not indicated" (if conventional tools or methods are superior), and "contraindicated" (only "if somebody uses the laser to solve his Ego problems"). Based on their experiences, the authors incorporated a pilot light to indicate to the surgeon the path and focus of the invisible $\mathrm{CO}_{2}$ laser beam, and developed a microadapter to maintain the laser at a fixed distance when working under the operating microscope (Fig. 5). In conclusion, they reflected that "after long years of a lonesome walk we have found today that many neurosurgeons throughout the whole world are in our company and using lasers." 2
Another large series by Takizawa, ${ }^{33}$ documented the use of the laser in 160 cases between 1969 and 1982, noting that the device allowed resections to be performed within the cavity of the tumor, limiting the amount of manipulation or retraction on adjacent structures. He remained more moderate about the role of the laser and remarked that adjuvant instruments, including the ultrasonic aspirator and bipolar and loop cautery, remained indispensable. He went on to comment that "the $\mathrm{CO}_{2}$ laser is nothing more than one of the surgical instruments, but it is no doubt a breakthrough in the surgery of brain tumours. Actually we have encountered many cases where extirpation of the tumour would have been impossible without the $\mathrm{CO}_{2}$ laser." In 1980 Beck $^{3}$ described 120 cases in which was used either the $\mathrm{CO}_{2}$ or Nd:YAG laser, noting the former had a better profile for cutting and reduced retraction in eloquent areas. In 1983 Edwards and coworkers ${ }^{9}$ reported the results of 80 neurosurgical procedures using the $\mathrm{CO}_{2}$ laser, noting that in 77 cases of mixed intra- and extraaxial cranial and spinal tumors, the laser was rated as "very helpful" or "helpful"; in just 2 cases of dorsal root entry zone lesions it was "not helpful," and in one axillary neuroma it was of "questionable benefit." After reporting that experimental lesions in cat brains showed less blood-brain barrier damage when made with the $\mathrm{CO}_{2}$ laser compared with bipolar cautery, Cozzens and Cerullo ${ }^{5}$ remarked that "in 1984, there is similar enthusiasm over the introduction and use of the carbon dioxide laser" as there had been over the introduction of the Bovie electrosurgical unit in 1930. Other developments reflecting the increasing role of lasers in surgery in general, and neurosurgery in particular, were the establishment of the journal Lasers in Surgery and Medicine in 1980 and the First American Congress on Lasers in Neurosurgery held in Chicago in 1981.

\section{Enthusiasm Tempered}

The increasing integration of computers and image guidance into the neurosurgical operating room in the middle to late 1980 s meant the rise of computer-assisted, stereotactic laser resection of tumors, pioneered by Kelly. ${ }^{20}$ Multiple stereotactic imaging modalities could be utilized to plan the trajectory for, and the resection of, a deep-seated tumor, with a laser attached to the microscope and driven by the computer, and the laser's progress tracked by the surgeon via a cursor on the screen. In 267 cases of deep intracranial tumors, Kelly reported $10 \%$ morbidity and $1 \%$ mortality rates while achieving aggressive gross-total resection of any tumor visible on imaging. One drawback to this method is the need for a relatively wide transparenchymal corridor to the tumor, which leads to significant collateral damage to neural tissue as opposed to other routes of access, and the technique is seldom used at present.

Endoscopic surgery allows minimally invasive approaches to numerous areas of the body, including an expanding number of intracranial locations. In 1970 Goodale et al. ${ }^{15}$ were the first to use a $\mathrm{CO}_{2}$ laser through a rigid endoscope to coagulate bleeding gastric ulcers. In 1973 Nath et al. ${ }^{25}$ reported the first use of a flexible fi- 
beroptic endoscope along with an argon laser. However, the long wavelength of the $\mathrm{CO}_{2}$ laser results in an excessive loss of power when transmitted through conventional solid fiberoptic cables, thus preventing its use in this manner. Although there have been reports on the use of argon lasers to fenestrate cysts via steerable endoscopes and a technique to use lasers during endoscopy in the ventricles via a ball tip that converts Nd:YAG light energy to thermal energy has been described, the serious risk of damage to adjacent structures by argon or Nd:YAG laser energy has limited their application in endoscopy. ${ }^{27,34,35}$

There is a wide range of opinions based on experience with laser systems, and it is likely that the ease of use and ability to manipulate the device are factors that determine whether the laser has utility in the neurosurgical operating room. In the most recent (1996) review of lasers in neurosurgery, Devaux and Roux ${ }^{7}$ have expressed their strong support for lasers, stating that these devices play a large role in the neurosurgical armamentarium. In sharp contrast, Laws commented on this review, downplayed the importance of the laser, and said, "I cannot think of a single case that I would cancel if the laser were broken and it is hard to remember the last time I used a laser in clinical surgery."7 Moreover, in reviewing operative techniques in neurosurgery, Rhoton ${ }^{28}$ describes lasers as useful for coagulation and debulking of tumors but personally prefers carefully applied bipolar cautery and microdissection for the removal of tumor adjacent to neural or vascular structures. Given the relative paucity of recent literature on lasers in neurosurgery, these tools do not currently seem to play a prominent role in the neurosurgical operating room. Cumbersome ergonomics, especially of the $\mathrm{CO}_{2}$ laser systems with bulky articulating arms, and the change in focal distance from attaching a microadapter for the laser to the operating microscope also deter their regular use. The large size of laser units (belied by comments from Stellar and Polanyi ${ }^{32}$ in 1970 , in an era when a computer could occupy an entire floor: "Suffice to say that this laser is compact, readily fitting into the laboratory or operating room") can also be problematic in modern operating rooms where space for equipment and personnel is at a premium. Finally, the perception that lasers can impede efficiency or slow down procedure time can also be seen as a likely barrier to their common use.

\section{Emerging Technology}

The development of a flexible, hollow-core fiber lined with an omnidirectional mirror for the delivery of $\mathrm{CO}_{2}$ laser energy may help to mitigate some of the above-described problems related to ergonomics. ${ }^{12,17}$ Recent laboratory and clinical experience suggests that it offers the low profile required for working under the microscope while bringing $\mathrm{CO}_{2}$ laser energy directly to the surgical site (Fig. 6). ${ }^{6}$ Other developments, such as combined $\mathrm{Nd}$ :YAG and $\mathrm{CO}_{2}$ lasers, may offer a single tool to take advantage of the hemostatic and cutting abilities of the 2 lasers. In addition, authors of numerous experiments, mostly in the laboratory but also in the clinical setting, continue to explore the functionality of the laser beyond photocoagulation and vaporization, including laser in- terstitial thermotherapy and photodynamic therapy for tumors, laser tissue welding such as laser-assisted nerve or vascular anastomosis, and laser discectomy. ${ }^{21}$ As the evidence of efficacy increases, well-designed trials will be required to determine the appropriate role of laser procedures in neurosurgical practice.

\section{Conclusions}

The utility of various lasers as neurosurgical instruments has been explored since shortly after the discovery of lasers. Experience has led to a refinement in the methods for delivering laser energy to the brain, and the enthusiasm for the use of lasers in the neurosurgical operating room has waxed and waned with these developments, from unbridled optimism to apathy. It seems likely that defining specific roles in which the laser offers clear benefit will be the next stage in their history as they secure a place in the neurosurgical armamentarium. The example of laser technology shows us that often there are excellent ideas that appear to have some innate practical neurosurgical worth, yet what seems to be a straightforward application is problematic or relies on advances in other scientific realms - such as material science in the case of lasers - to become a truly successful technology.

\section{Disclaimer}

The authors report no conflict of interest concerning the materials or methods used in this study or the findings specified in this paper.

\section{References}

1. Ascher PW: Newest ultrastructural findings after the use of a $\mathrm{CO}_{2}$-laser on CNS tissue. Acta Neurochir Suppl (Wien) 28: 572-581, 1979

2. Ascher PW, Heppner F: $\mathrm{CO}_{2}$-laser in neurosurgery. Neurosurg Rev 7:123-133, 1984

3. Beck OJ: The use of the Nd-YAG and the $\mathrm{CO}_{2}$ laser in neurosurgery. Neurosurg Rev 3:261-266, 1980

4. Brown TE, True C, Mclaurin RL, Hornby P, Rockwell RJ: Craniocerebral trauma induced by laser radiation. A method for experimental production of subdural and subarachnoid hematoma. Life Sci 5:81-87, 1966

5. Cozzens JW, Cerullo LJ: Comparison of the effect of the carbon dioxide laser and the bipolar coagulator on the cat brain. Neurosurgery 16:449-453, 1985

6. Devaiah AK, Shapshay SM, Desai U, Shapira G, Weisberg O, Torres DS, et al: Surgical utility of a new carbon dioxide laser fiber: functional and histological study. Laryngoscope 115:1463-1468, 2005

7. Devaux BC, Roux FX: Experimental and clinical standards, and evolution of lasers in neurosurgery. Acta Neurochir (Wien) 138:1135-1147, 1996

8. Earle KM, Carpenter S, Roessmann U, Ross MA, Hayes Jr, Zeitler E: Central nervous system effects of laser radiation. Fed Proc (24 Suppl):S129-S139, 1965

9. Edwards MS, Boggan JE, Fuller TA: The laser in neurological surgery. J Neurosurg 59:555-566, 1983

10. Eggert HR, Kiessling M, Kleihues P: Time course and spatial distribution of neodymium: yttrium-aluminum-garnet (Nd:YAG) laser-induced lesions in the rat brain. Neurosurgery 16:443-448, 1985

11. Fine S, Klein E, Nowak W: Interaction of laser radiation with biologic systems. I. Studies on interaction with tissues. Fed Proc (14 Suppl):S35-S45, 1965 


\section{R. W. Ryan, R. F. Spetzler, and M. C. Preul}

12. Fink Y, Winn JN, Fan S: A dielectric omnidirectional reflector. Science 282:1679-1682, 1998

13. Fox JL, Hayes JR, Stein MN, Green RC, Paananen R: Experimental cranial and vascular studies of the effects of pulsed and continuous wave laser radiation. J Neurosurg 27:126137, 1967

14. Fuller TA: The physics of surgical lasers. Lasers Surg Med 1:5-14, 1980

15. Goodale RL, Okada A, Gonzales R, Borner JW, Edlich RF, Wangensteen $\mathrm{OH}$ : Rapid endoscopic control of bleeding gastric erosions by laser radiation. Arch Surg 101:211-214, 1970

16. Hall RR, Beach AD, Baker E, Morison PC: Incision of tissue by carbon dioxide laser. Nature 232:131-132, 1971

17. Hart SD, Maskaly GR, Temelkuran B, Prideaux PH, Joannopoulos JD, Fink Y: External reflection from omnidirectional dielectric mirror fibers. Science 296:510-513, 2002

18. Jain KK: Lasers in neurosurgery: a review. Lasers Surg Med 2:217-230, 1983

19. Javan A, Bennett WR, Herriott DR: Population inversion and continuous optical maser oscillation in a gas discharge containing a He-Ne mixture. Phys Rev Lett 6:106-110, 1961

20. Kelly PJ: Future perspectives in stereotactic neurosurgery: stereotactic microsurgical removal of deep brain tumors. J Neurosurg Sci 33:149-154, 1989

21. Krishnamurthy S, Powers SK: Lasers in neurosurgery. Lasers Surg Med 15:126-167, 1994

22. Maiman TH: Stimulated optical radiation in ruby. Nature 187:493-494, 1960

23. McGuff PE, Deterling RA Jr, Gottlieb LS, Fahimi HD, Bushnell D: Surgical applications of laser. Ann Surg 160:765-777, 1964

24. Minton JP, Ketcham AS: The effect of ruby laser radiation on the Cloudman S-91 melanoma in the CDBA-2F hybrid mouse. Cancer 17:1305-1309, 1964

25. Nath G, Gorisch W, Kiefhaber P: First laser endoscopy via a fiberoptic transmission system. Endoscopy 5:208-213, 1973
26. Patel CKN: Continuous-wave laser action on vibrational-rotational transitions of $\mathrm{CO}_{2}$. Phys Rev 136A:1187-1193, 1964

27. Powers SK: Fenestration of intraventricular cysts using a flexible, steerable endoscope and the argon laser. Neurosurgery 18:637-641, 1986

28. Rhoton AL Jr: Operative techniques and instrumentation for neurosurgery. Neurosurgery 53:907-934, 2003

29. Rosomoff HL, Carroll F: Effect of laser on brain and neoplasm. Surg Forum 16:431-433, 1965

30. Rosomoff HL, Carroll F: Reaction of neoplasm and brain to laser. Arch Neurol 14:143-148, 1966

31. Stellar S, Polanyi TG: Lasers in neurosurgery: a historical overview. J Clin Laser Med Surg 10:399-411, 1992

32. Stellar S, Polanyi TG, Bredemeier HC: Experimental studies with the carbon dioxide laser as a neurosurgical instrument. Med Biol Eng 8:549-558, 1970

33. Takizawa T: The carbon dioxide laser surgical unit as an instrument for surgery of brain tumours-its advantages and disadvantages. Neurosurg Rev 7:135-144, 1984

34. Van Beijnum J, Hanlo PW, Fischer K, Majidpour MM, Kortekaas MF, Verdaasdonk RM, et al: Laser-assisted endoscopic third ventriculostomy: long-term results in a series of 202 patients. Neurosurgery 62:437-443, 2008

35. Vandertop WP, Verdaasdonk RM, van Swol CF: Laser-assisted neuroendoscopy using a neodymium-yttrium aluminum garnet or diode contact laser with pretreated fiber tips. J Neurosurg 88:82-92, 1998

Manuscript submitted May 18, 2009.

Accepted June 30, 2009.

Address correspondence to: Mark C. Preul, M.D., Neurosurgery Research Laboratory, Barrow Neurological Institute, St. Joseph's Hospital and Medical Center, 350 West Thomas Road, Phoenix, Arizona, 85013. email: Mark.Preul@chw.edu. 